University of Wollongong

Research Online

Australian Institute for Innovative Materials -

Papers

Australian Institute for Innovative Materials

January 2019

On Low-Concentration Inks Formulated by Nanocellulose Assisted with Gelatin Methacrylate (GeIMA) for 3D Printing toward Wound Healing Application

\author{
Wenyang $\mathrm{Xu}$ \\ Abo Akademi University \\ Binbin Molino \\ University of Wollongong \\ Fang Cheng \\ Sun Yat-Sen University \\ Paul J. Molino \\ University of Wollongong, pmolino@uow.edu.au \\ Zhilian Yue \\ University of Wollongong, zyue@uow.edu.au
}

See next page for additional authors

Follow this and additional works at: https://ro.uow.edu.au/aiimpapers

Research Online is the open access institutional repository for the University of Wollongong. For further information contact the UOW Library: research-pubs@uow.edu.au 


\title{
On Low-Concentration Inks Formulated by Nanocellulose Assisted with Gelatin Methacrylate (GeIMA) for 3D Printing toward Wound Healing Application
}

\author{
Abstract \\ Cellulose nanofibrils (CNFs) in the form of hydrogels stand out as a platform biomaterial in bioink \\ formulation for 3D printing because of their low cytotoxicity and structural similarity to extracellular \\ matrices. In the present study, 3D scaffolds were successfully printed with low-concentration inks \\ formulated by $1 \mathrm{w} / \mathrm{v} \%$ 2,2,6,6-Tetramethylpiperidine-1-oxyl radical (TEMPO)-oxidized CNF with less than 1 \\ $\mathrm{w} / \mathrm{v} \%$ gelatin methacrylate (GelMA). Quartz crystal microbalance with dissipation monitoring (QCM-D) \\ measurements showed strong interaction between the two biopolymers. The UV cross-linking ability of \\ GelMA ( $\leq 1 \mathrm{w} / \mathrm{v} \%$ ) was enhanced in the presence of TEMPO-oxidized CNFs. Multiple factors including \\ strong physical interaction between CNF and GeIMA, in situ cross-linking of CNF by Ca 2+ , and UV cross- \\ linking of GelMA enabled successful 3D printing of low-concentration inks of CNF/GelMA into scaffolds \\ possessing good structural stability. The mechanical strength of the scaffolds was tuned in the range of \\ 2.5 to $5 \mathrm{kPa}$. The cell culture with 3T3 fibroblasts revealed noncytotoxic and biocompatible features for \\ the formulated inks and printed scaffolds. More importantly, the incorporated GelMA in the CNF hydrogel \\ promoted the proliferation of fibroblasts. The developed low-concentration CNF/GelMA formulations with \\ a facile yet effective approach to fabricate scaffolds showed great potential in 3D printing for wound \\ healing application.

\section{Publication Details} \\ Xu, W., Molino, B. Zhang., Cheng, F., Molino, P. J., Yue, Z., Su, D., Wang, X., Willfor, S., Xu, C. \& Wallace, G. G. \\ (2019). On Low-Concentration Inks Formulated by Nanocellulose Assisted with Gelatin Methacrylate \\ (GeIMA) for 3D Printing toward Wound Healing Application. ACS Applied Materials and Interfaces, 11 (9), \\ 8838-8848.
}

\section{Authors}

Wenyang Xu, Binbin Molino, Fang Cheng, Paul J. Molino, Zhilian Yue, Dandan Su, Xiaoju Wang, Stefan Willfor, Chunlin Xu, and Gordon G. Wallace 


\section{On Low-Concentration Inks Formulated by Nanocellulose Assisted with Gelatin Methacrylate (GeIMA) for 3D Printing toward Wound Healing Application}



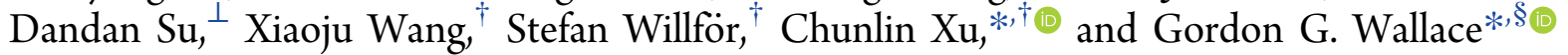

${ }^{\dagger}$ Laboratory of Wood and Paper Chemistry, Johan Gadolin Process Chemistry Centre, Åbo Akademi University, Porthansgatan 3, 20500 Turku, Finland

${ }^{\S}$ ARC Centre of Excellence for Electromaterials Science, Intelligent Polymer Research Institute, University of Wollongong,

Wollongong, NSW 2522, Australia

"Faculty of Engineering, Yokohama National University, Yokohama 240-8501, Japan

${ }^{\perp}$ School of Pharmaceutical Sciences (Shenzhen), Sun Yat-sen University, 510006 Guangzhou, China

${ }^{\ddagger}$ Cell Biology, Faculty of Science and Engineering, Åbo Akademi University, Tykistökatu 6, 20520 Turku, Finland

Supporting Information

ABSTRACT: Cellulose nanofibrils (CNFs) in the form of hydrogels stand out as a platform biomaterial in bioink formulation for 3D printing because of their low cytotoxicity and structural similarity to extracellular matrices. In the present study, $3 \mathrm{D}$ scaffolds were successfully printed with low-concentration inks formulated by 1 w/v \% 2,2,6,6-tetramethylpiperidine-1-oxyl radical (TEMPO)-oxidized CNF with less than $1 \mathrm{w} / \mathrm{v} \%$ gelatin methacrylate (GelMA). Quartz crystal microbalance with dissipation monitoring (QCM-D) measurements showed strong interaction between the two biopolymers. The UV cross-linking ability of GelMA $(\leq 1 \mathrm{w} / \mathrm{v}$ $\%)$ was enhanced in the presence of TEMPO-oxidized CNFs. Multiple factors including strong physical interaction between CNF and GelMA, in situ crosslinking of CNF by $\mathrm{Ca}^{2+}$, and UV cross-linking of GelMA enabled successful 3D printing of low-concentration inks of CNF/GelMA into scaffolds possessing good structural stability. The mechanical strength of the scaffolds was tuned in the range

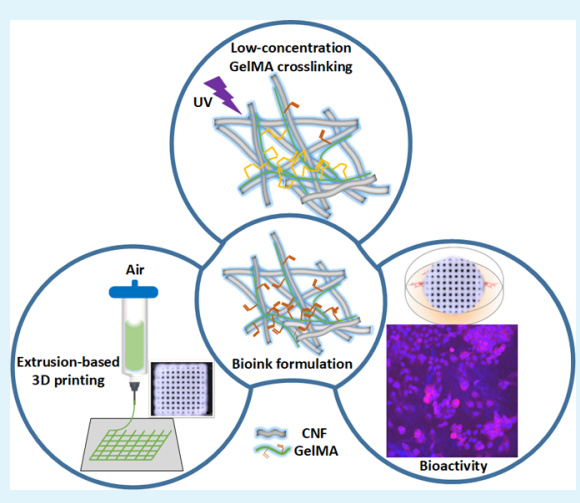
of 2.5 to $5 \mathrm{kPa}$. The cell culture with $3 \mathrm{~T} 3$ fibroblasts revealed noncytotoxic and biocompatible features for the formulated inks and printed scaffolds. More importantly, the incorporated GelMA in the CNF hydrogel promoted the proliferation of fibroblasts. The developed low-concentration CNF/GelMA formulations with a facile yet effective approach to fabricate scaffolds showed great potential in $3 \mathrm{D}$ printing for wound healing application.

KEYWORDS: Low-concentration ink formulation, Cellulose nanofibrils (CNFs), Gelatin methacrylate (GelMA), UV cross-linking, $3 D$ printing, Wound healing

\section{INTRODUCTION}

Three-dimensional (3D) printing allows the patterning of soft biocompatible hydrogels into 3D tissue that mimic constructs, generating intricately structured scaffolds that permit researchers to study cell-cell and cell-matrix interactions. ${ }^{1,2}$ Cellulose nanomaterials, including cellulose nanocrystals $(\mathrm{CNCs})^{3-7}$ and cellulose nanofibrils (CNFs), have attracted increasing attention as bioink constituents for $3 \mathrm{D}$ bioprinting. ${ }^{8,9}$ This is attributable to their low cytotoxicity, structural similarity to extracellular matrices (ECM), and favorable rheological properties. As the pioneer in this field, Gatenholm's group has largely contributed to the development of nanocellulosebased bioinks from 2015 for different applications including cartilage regeneration, human chondrocytes redifferentiation, and adipose tissue engineering. ${ }^{10-16}$
As required in extrusion-based hydrogel printing, the rheological property (i.e., flow behavior) of a well-defined bioink must show shear thinning and thixotropic behaviors, which is consistent with the nature of nanocellulose. ${ }^{3,17-19}$ Still, the most challenging aspect is to achieve good structural stability of the printed constructs, particularly for the fabrication of objects with complex geometries. Therefore, an auxiliary material is preferable to be incorporated in CNFbased bioink formulation, which imposes functionalities to aid printability and good fidelity, as well as to increase ink bioactivity. ${ }^{8,9}$ Common strategies include direct or indirect solidification of printed objects by cross-linking either

Received: December 4, 2018

Accepted: February 8, 2019

Published: February 11, 2019 
nanocellulose $\mathrm{e}^{20,21}$ or the incorporated auxiliary materials. The auxiliary materials can be natural or synthetic polymers that are amenable to ionic cross-linking, ${ }^{16}$ photochemical cross-linking, ${ }^{4}$ thermal cross-linking, ${ }^{13}$ or as a sacrificial supporter. ${ }^{22}$

Gelatin, a derivative of collagen, resembles the biological structure of collagen in the native ECM tissues. Gelatin retains its natural cell binding motifs, such as RGD peptides, which improve cell responses such as cell adhesion, proliferation, migration, and differentiation. ${ }^{23,24}$ Gelatin together with other biopolymers such as alginate, ${ }^{25}$ collagen, ${ }^{26}$ and hyaluronic $\operatorname{acid}^{27}$ has been formulated into hydrogel bioinks for assessment in various biomedical applications. Moreover, the methacrylated gelatin (GelMA) with favorable thermal sensitivity and photo-cross-linking ability has been broadly evaluated in bioink formulations. ${ }^{28-30}$ Low-concentration GelMA (i.e., $\leq 5 \%$ ) bioinks are attractive because of their relatively loose polymer network that can encourage cell-cell interaction, migration, and more efficient metabolism. Recently, Yin et al. successfully established a two-step crosslinking strategy for bioprinting of 5-30 w/v \% GelMA assisted with thermal-sensitive gelatin. ${ }^{31}$ Shin et al. have reported 3D printing of $5 \mathrm{w} / \mathrm{v} \%$ GelMA by tuning its rheological property with different concentrations of mechanically grinded CNF suspensions. The interconnected pore network established by such hydrogels benefits the cell viability and supports cell proliferation as well. ${ }^{32}$

The approach used in the present study employs an extremely low concentration of GelMA $(\leq 1 \mathrm{w} / \mathrm{v} \%)$ as the auxiliary material for the TEMPO-mediated oxidized CNF based bioink. Herein, the interaction between negatively charged CNF and GelMA could not only regulate the viscosity of the formulated bioinks but also facilitate the cross-linking of low-concentration GelMA ( $\leq 1 \mathrm{w} / \mathrm{v} \%)$. First, the bioinks of CNFs and GelMA were formulated and optimized through rheological studies and QCM-D measurements. Well-defined and freestanding 3D hydrogel scaffolds of CNF/GelMA in high resolutions were successfully fabricated via an extrusionbased printing technique. Finally, the cytotoxicity and bioactivity of the CNF/GelMA bioinks were assessed by cell viability and cell proliferation of $3 \mathrm{~T} 3$ fibroblasts.

\section{MATERIALS AND METHODS}

Materials. TEMPO-oxidized CNF (CNF) $(1.0 \mathrm{w} / \mathrm{v} \%)$ with moderate charge density $(1.14 \pm 0.07 \mathrm{mmol} / \mathrm{g})$ was produced by TEMPO $/ \mathrm{NaClO} / \mathrm{NaBr}$ oxidation according to Liu et al. ${ }^{33}$ Gelatin (porcine skin, type A, bloom number 300) was purchased from Sigma-Aldrich. GelMA (DS $70 \pm 8 \%$ ) was produced from a reaction with methacrylic anhydride. ${ }^{24,34} \mathrm{CaCl}_{2}$ and 2-hydroxy-1-(4-(2hydroxyethoxy)phenyl)-2-methyl-1-propanone (Irgacure 2959) were purchased from Sigma-Aldrich. PBS with $0.9 \mathrm{mM} \mathrm{CaCl}_{2}$ and $0.5 \mathrm{mM}$ $\mathrm{MgCl}_{2}$ was purchased from Sigma-Aldrich and used in all the studies except cell studies.

Bioink Formulation. Bioink formulation was prepared using two methods. In the first method, dried GelMA powder was mixed with the CNF hydrogel $(1.0 \mathrm{w} / \mathrm{v} \%)$ at $50{ }^{\circ} \mathrm{C}$ using a vortex mixer (FINEPCR, Korea) until GelMA was completely dissolved to produce a homogeneous ink. Scheme 1 illustrates the procedure. The compositional ratios between CNF and GelMA were 5:1 and 2:1 $\mathrm{w} / \mathrm{w}$ (Table 1). The second method involved loading of $1 \mathrm{~mL}$ of 10 $\mathrm{w} / \mathrm{v} \%$ GelMA solution into $9 \mathrm{~mL}$ of CNF hydrogel to form homogeneous ink CNF:GelMA=9:10. Irgacure 2959 was selected as the photoinitiator and added to each ink with a final concentration of $0.5 \mathrm{w} / \mathrm{v} \%$.

Scaffold Fabrication. Scaffolds were fabricated with a customized extrusion-based 3D printer KIMM SPS1000 Bioplotter (Machtronics
Scheme 1. Schematic Illustration of Bioink Formulation and Scaffold Printinga ${ }^{a}$



${ }^{a}$ From left to right: images of GelMA and CNF hydrogel and their formulation process; simple illustration of direct ink writing (DIW) printing working principle; optical microscopic images of printed hydrogel scaffolds.

4 Technology, Korea). The KIMM Bioplotter software was used to produce a G-code file for printing. A 3D scaffold model with dimensions of $10 \mathrm{~mm}$ wide, $10 \mathrm{~mm}$ long, and 2 or $3 \mathrm{~mm}$ high was designed with a $1 \mathrm{~mm}$ filament grid and $0.2 \mathrm{~mm}$ layer thickness. The formulated bioinks were transferred to a 5 cc EFD syringe (Optimum by Nordson EFD, USA), which was controlled by a pneumatic dispensing head with a pressure regulator $(\mathrm{AD}$ 3000C, Iwashita Engineering, Japan). Precision tips (25 and 30 GA, Nordson EFD, USA) were used as the dispensing nozzles. $5 \% \mathrm{CaCl}_{2}$ solution was added dropwise on the substrate to stabilize the printed structure. UV $(365 \mathrm{~nm})$ irradiation with an intensity of $10 \mathrm{~mW} / \mathrm{cm}^{2}$ for 5 min was applied to cross-link GelMA. The investigated printing parameters of pressure and speed are listed in Table 1.

Characterization. Rheology. Rheology studies were carried out on a Physica MCR 301 rheometer (Anton-Paar, Austria) with a coneplate geometry $\left(\varnothing 50 \mathrm{~mm}\right.$ and $\left.1^{\circ}\right)$. The viscosity property was recorded with shear rates of $0.01-100 \mathrm{~s}^{-1}$ at room temperature. Oscillation measurements were performed on the inks under a temperature linear ramp of 40 to $4{ }^{\circ} \mathrm{C}$ with constant strain at $1 \%$ and sweep at $1.5 \mathrm{~Hz}$. Yield stress of the inks was tested with varying shear stress from 1 to $100 \mathrm{~Pa}$. Storage and loss modulus during UV gelation were tested under UV intensity at $10 \mathrm{~mW} / \mathrm{cm}^{2}$.

Quartz Crystal Microbalance with Dissipation Monitoring (QCM-D). The adsorption of GelMA on CNF was studied by QCM-D using a Q-Sense E4 instrument (Q-sense, Västra Frölunda, Sweden) according to the modified method from Österberg et al., 35,36 CNF dispersion was prepared by diluting the CNF hydrogel $(1.0 \mathrm{w} / \mathrm{v}$ $\%$ dry matter content) with Milli-Q water followed by ultrasonication at $25 \%$ amplitude for 5 min using a Branson Digital Sonifier 450 (Branson Corporation, Danbury, CT). The diluted CNF dispersion was then centrifuged at $8000 \mathrm{rpm}$ for $30 \mathrm{~min}$ at room temperature with a PrO-Research centrifuge (Centurion Scientific Ltd.) to separate CNF fibrils from larger fibril bundles. The supernatant fraction with the finest CNF fibrils (about $1.25 \mathrm{mg} / \mathrm{mL}$ dry matter content) was collected for further use.

In order to enhance retention of CNF, polyethylene imine (PEI) was preadsorbed on the surface of the gold-coated quartz crystals by passing an aqueous PEI solution with a concentration of $0.2 \mathrm{mg} / \mathrm{mL}$ through the QCM-D measurement chamber for $20 \mathrm{~min}$, followed by rinsing with DI water. Diluted CNF dispersion was then injected into the QCM-D chamber for adsorption of the CNF layer onto the PEImodified QCM-D sensor for 20 min followed by rinsing with DI water. Thereafter, the CNF-modified sensors were exposed to GelMA solutions with gradient concentrations (based on weight ratios of $1: 2$, 1:5, and 10:9 relative to dry content of CNF). The adsorption of a 0.5 $\mathrm{w} / \mathrm{v} \%$ gelatin solution was also tested. The flow rate through the 
Table 1. Formulation and Printing Parameters for CNF/GelMA Bioinks

\begin{tabular}{|c|c|c|c|c|c|c|}
\hline ink code & $\mathrm{CNF}, \mathrm{w} / \mathrm{v} \%$ & GelMA, w/v \% & pressure, $\mathrm{kPa}$ & speed, $\mathrm{mm} \mathrm{min}^{-1}$ & theoretical diameter, $\mathrm{mm}$ & real diameter, $\mathrm{mm}$ \\
\hline $\mathrm{CNF}^{a}$ & 1 & & 70 & 1000 & 0.25 & $0.59 \pm 0.05$ \\
\hline CNF:GelMA $=5: 1^{b}$ & 1 & 0.2 & 65 & 2000 & 0.24 & $0.45 \pm 0.03$ \\
\hline CNF:GelMA $=2: 1^{b}$ & 1 & 0.5 & 70 & 2000 & 0.26 & $0.57 \pm 0.07$ \\
\hline CNF:GelMA $=9: 10^{c}$ & 0.9 & 1 & 80 & 2000 & 0.25 & $0.60 \pm 0.04$ \\
\hline
\end{tabular}

${ }^{a}$ Printing tips with $27 \mathrm{G}$ were used. ${ }^{b}$ Dry powder of GelMA was mixed with $1 \mathrm{w} / \mathrm{v} \%$ CNF hydrogel, resulting in 0.2 and $0.5 \mathrm{w} / \mathrm{v} \%$ final GelMA concentrations, respectively, and printing tips with $30 \mathrm{G}$ were used. ${ }^{c} 10 \mathrm{w} / \mathrm{v} \%$ GelMA solution added to $1 \mathrm{w} / \mathrm{v} \% \mathrm{CNF}$, resulting in $1 \mathrm{w} / \mathrm{v} \%$ GelMA final concentration and $0.9 \mathrm{w} / \mathrm{v} \% \mathrm{CNF}$ final concentration, and printing tips with $30 \mathrm{G}$ were used.

QCM-D chambers was set to $40 \mu \mathrm{L} / \mathrm{min}$ and kept constant during measurements.

Both the frequency and dissipation parameters were recorded during the adsorption of material to the QCM-D sensor surface. The significant positive shift in the dissipation parameter, relative to the frequency, on adsorption of GelMA and gelatin to the sensor surface is representative of the adsorption of a soft viscoelastic surface layer, and therefore, a viscoelastic model is required to appropriately characterize the material properties. Therefore, the Voigt model was applied to the QCM-D data set using the Q-Sense QTools analysis software Version 3.0.10.286 (Biolin Scientific AB). All experiments were run in triplicate. The following input parameters within the model provided the best fit for the layer density $\left(1150 \mathrm{~kg} / \mathrm{m}^{3}\right)$, fluid density $\left(1020 \mathrm{~kg} / \mathrm{m}^{3}\right)$, layer viscosity $\left(10^{-6} \leq 10^{-2} \mathrm{~kg} / \mathrm{ms}\right)$, layer shear modulus $\left(10^{4} \leq 10^{8} \mathrm{~Pa}\right)$, and mass $\left(1.15 \leq 1.15^{5} \mathrm{ng} / \mathrm{cm}^{2}\right)$. The third, fifth, and seventh overtones were used for modeling calculations. According to the Sauerbrey equation (eq 1), the change in frequency $(\Delta f)$ is proportional to the mass adsorbed per unit surface $(\Delta m)$

$$
\Delta m=\frac{-C \Delta f}{n}
$$

where $C$ is the sensitivity constant (here, $C=0.177 \mathrm{mg} / \mathrm{m}^{2}$ ) and $n$ is the overtone number (here, $n=3$ ). Equation 1 is valid for thin, rigid, and uniform films, but it underestimates mass for viscoelastic films, when dissipation $\Delta D$ is $>1 .^{37}$ Therefore, the calculated mass values presented are estimations and should not be considered as absolute values.

Mechanical Tests. Compressive strength of the printed scaffolds was measured with a Shimadzu EZ-L universal testing machine with a $10 \mathrm{~N}$ load cell controlled by the TRAPEZIUMX software. The compression speed was set with a constant rate of $0.5 \mathrm{~mm} / \mathrm{min}$. Compressive Young's modulus was calculated using eq 2

$$
E=\frac{F \times L_{0}}{A \times \Delta L}
$$

where $F(\mathrm{~N})$ is the recorded compressive force, $L_{0}(\mathrm{~mm})$ is the original height of the scaffold, $A$ is the area of the scaffold in contact with the upper compression plate (here, the scaffold with solid surface of $10 \times 10 \mathrm{~mm}$ was used), and $\Delta L$ is set with a constant displacement of $2 \mathrm{~mm}$.

Cast disc samples were also prepared for compression tests. The uniform discs were cast by first injecting formulated inks into an 8.0 $\mathrm{mm}$ disc mold followed by adding $\mathrm{CaCl}_{2}$ solution for ionic crosslinking; the cross-linked discs were then exposed to $5 \mathrm{~min}$ of UV irradiation. The cast discs were stored in $\mathrm{PBS} / \mathrm{Ca}^{2+}$ overnight prior to compression tests.

AFM Surface Young's Modulus Measurements. The surface Young's moduli of $3 \mathrm{D}$ printed scaffolds of either CNF or CNF/ GelMA scaffolds were measured using a JPK Nanowizard II AFM (JPK Instruments AG, Berlin, Germany). Force measurements were performed in PBS using a DNP-S10 silicon nitride cantilever (spring constant $\sim 0.06 \mathrm{~N} \mathrm{~m}^{-1}$ ) with a $10 \mathrm{~nm}$ round tip. The spring constant of each cantilever was calibrated using the thermal method, and the deflection sensitivity was determined by performing a force measurement on a clean glass slide in PBS prior to undertaking measurements on the scaffolds. Force measurements were performed at a minimum of three different locations on each scaffold, with five separate measurements taken at each location and averaged. Three separate scaffolds were analyzed for each material.

Swelling Tests. The printed hydrogel scaffolds were washed with Milli-Q water to remove the excess $\mathrm{Ca}^{2+}$ and then frozen by liquid $\mathrm{N}_{2}$ prior to freeze-drying. When the freeze-dried scaffolds were rehydrated with Milli- $\mathrm{Q}$ water, the gravimetrical water uptake was measured after the excess water on the surface was carefully removed with tissue paper. Then, the swelling ratio was calculated according to eq 3.

$$
\text { swelling ratio }(\mathrm{g} / \mathrm{g})=\frac{m_{\mathrm{wet}}-m_{\mathrm{dry}}}{m_{\mathrm{dry}}}
$$

Optical Microscopy and Scanning Electron Microscopy. The microscopic images of the printed scaffolds were observed using a LEICA M205A optical microscope. The filament diameter was calculated on the average of 20 filaments among the printed scaffolds. Morphological features of the scaffold strut were characterized by scanning electron microscopy (SEM) after freeze-drying.

Quantitative Cell Viability MTT Assay and Cell Attachment Assay. 3T3 fibroblast cells, which were isolated from E12 SVJ129 mouse embryos to establish 3T3 cells in the lab, were maintained with DMEM (4.5 mM glucose) supplemented with $2 \mathrm{mM}$ L-glutamine, 100 $\mathrm{IU} / \mathrm{mL}$ penicillin and streptomycin, and $10 \%$ heat-inactivated FBS (Invitrogen) and were incubated with $5 \% \mathrm{CO}_{2}$ at $37{ }^{\circ} \mathrm{C}$ and $95 \%$ humidity. The $2 \mathrm{D}$ control cells are the cells growing on coverslips. After splitting, the initial cell seeding density was $1 \times 10^{5}$ cells $/ 1.9$ $\mathrm{cm}^{2}$, and once the cell suspension had been in contact with the matrix for $12 \mathrm{~h}$, nonadherent cells were washed off and the plate were frozen for $30 \mathrm{~min}$ at $-70{ }^{\circ} \mathrm{C}$. The 24 well plates were then thawed, and cells were stained in $0.5 \%$ crystal violet dye for $20 \mathrm{~min}$. After washing, samples were fixed with methanol for $20 \mathrm{~min}$, and then the optical density of each well was determined at $570 \mathrm{~nm}\left(\mathrm{OD}_{570}\right)$ with the microplate reader. Similarly, cytotoxicity evaluation of the 3D matrix was performed after $24 \mathrm{~h}$ of cell incubation using the MTT kit (Sigma) according to the supplier's manual. The optical density of each well in 24 well plates was immediately measured at $570 \mathrm{~nm}$ using the microplate reader and with wavelength higher than $650 \mathrm{~nm}$ to subtract the background.

Confocal Image Analysis of Cells on the Scaffolds. Cells were incubated with the 3D matrix in the cell culture medium for $72 \mathrm{~h}$. Then, cell samples were fixed with $3.7 \%$ paraformaldehyde for $30 \mathrm{~min}$ followed by a mixture of acetone and methanol (1:1) for $5 \mathrm{~min}$ in ice. Triton X $(0.2 \%)$ in PBS was used to permeabilize the cell membranes, and 10\% FBS in Triton X-100 and PBS was used for blocking for $2 \mathrm{~h}$. The cells were stained with Phalloidin conjugated with Alexa Fluor 546 for $3 \mathrm{~h}$ and counterstained with DAPI blue for $5 \mathrm{~min}$ before being mounted onto the glass slides. Confocal images were acquired at room temperature using Zeiss Zen software on a Zeiss LSM780 confocal laser scanning microscope (Carl Zeiss, Inc.) with PlanApochromat 20x. The following fluorochromes were used: Alexa Fluor 546 and 405.

Statistical Analysis. The results are expressed as mean \pm STDEV. Comparisons between two groups were analyzed by two-tailed $t$ tests. Comparisons between multiple groups were analyzed by one-way ANOVA. $P<0.05$ was considered significant. Statistical differences 



Figure 1. QCM-D analysis: (a) adsorption of GelMA onto CNF model layer with GelMA concentration from 0.2 to $1.0 \mathrm{w} / \mathrm{v} \%$ followed by desorption of free GelMA with rinsing DI water and the adsorption of a $0.5 \mathrm{w} / \mathrm{v} \%$ gelatin solution was also tested; (b) amounts of adsorbed CNF on the crystal and adsorbed GelMA on the CNF layer. Rheological analysis: (c) shear-thinning property of the formulated bioinks of CNF hydrogel $(1.0 \mathrm{w} / \mathrm{v} \%)$ and CNF/GelMA hydrogels with weight compositional ratios of 5:1, 2:1, and 9:10. (d) Change of storage moduli of the formulated bioinks after UV irradiation under oscillatory analysis indicating efficient and effective UV cross-linking; UV was switched on at $120 \mathrm{~s}$ and continued for $6 \mathrm{~min}$.

were calculated with the two-tailed unpaired $t$ test, and differences were considered significant at $p \leq 0.05$.

\section{RESULTS AND DISCUSSION}

Bioink Formulation: Molecular Interaction Characterized by QCM-D and Ink Rheological Property. To achieve good printability, the formulated inks are required to be homogeneous and extrudable with a consistent flow without clogging while printing. CNFs in this study were produced by the pretreatment of TEMPO-mediated oxidation followed by high-pressure homogenization. Thus, the dominating nanofibers in the inks had narrow size distribution in terms of fiber length, ${ }^{33}$ which resulted in a successful printing process without needle clogging. The density of charge introduced by TEMPO-mediated oxidation plays a decisive role in the disintegration of fibers to prepare nanodimensional fibrils. However, it was found out that a relatively lower surface charge density could better facilitate cell growth and proliferation. ${ }^{38}$ Thus, a negative surface charge in a density of $1.14 \pm 0.07$ $\mathrm{mmol} / \mathrm{g}$ was introduced on the fiber surface with $-\mathrm{COO}^{-}$ during the TEMPO-mediated oxidation. Meanwhile, the GelMA is positively charged when the ink is formulated under neutral conditions ( $\mathrm{pK} K_{\mathrm{a}}$ around 9$) .{ }^{39-41}$ Thus, the ionic interaction between two biopolymers is anticipated. To achieve ink homogeneity, the concentration of GelMA was screened from $5 \mathrm{w} / \mathrm{v} \%$ down to a maximum of $1 \mathrm{w} / \mathrm{v} \%$ to avoid phase separation caused by ionic interaction between CNF and GelMA.

It is crucial to understand the macromolecular interactions between CNF and GelMA in order to study the physiochemical properties of the formulated bioink. Therefore, the interaction between GelMA and the CNF layer was studied in situ with the QCM-D technique in aqueous media. As indicated by the adsorption kinetics of various concentrations of GelMA on CNF (presented in Figure 1a), the GelMA was strongly adsorbed onto the CNF layer. As estimated from the Sauerbrey equation, the absorption mass of GelMA went up from 100 to $150 \mathrm{mg} / \mathrm{m}^{2}$ when the concentration of GelMA increased from 0.2 to $0.5 \mathrm{w} / \mathrm{v} \%$. As shown in Figure $1 \mathrm{~b}$, the adsorption amount became less significant with further increases in the GelMA concentration, suggesting that the CNF layer became saturated with GelMA. This is correlated with the findings that the formulation of bionks using concentrations of GelMA of less than $1 \mathrm{w} / \mathrm{v} \%$ could avoid phase separation. As previously studied in the adsorption of various biopolymers on $\mathrm{CNF}^{36,42,43}$ strong hydrogen bonding 
and physical entanglement mainly contribute to the adsorption. In this case, the high adsorption mass amount and strong affinity of GelMA to CNF are probably associated with ionic interactions in addition to hydrogen bonding between both macromolecules. ${ }^{39}$ Moreover, $0.5 \mathrm{w} / \mathrm{v} \%$ gelatin was also used in the QCM-D measurement as a comparison with GelMA. It showed much less adsorption compared with GelMA solutions (Figure 1a,b), which indicated that the methacryloylation of gelatin had an impact in an interactive manner between the gelatin molecule and the CNF surface, possibly due to the hydrophobic nature and steric effect of the grafted methacryloyls.

The rheological behaviors of the formulated bioinks were assessed to validate their feasibility for $3 \mathrm{D}$ printing. The viscosity properties of CNF/GelMA bioinks as investigated are shown in Figure 1c. Also studied by other researchers, ${ }^{16,20}$ CNF itself showed a shear-thinning behavior, which is required for the extrusion-based printing process. By incorporating GelMA into the CNF hydrogel, similar yield-flow behaviors were registered for all the formulated inks. However, the zeroshear viscosity plateau was prolonged with the addition of more GelMA, as it might require a longer time for the polymer chains to orient in the formulated bioinks. Meanwhile, the zero-shear viscosity of the ink decreased with the incorporation of GelMA because of the physiochemical interactions between the two macromolecules, which is consistent with the QCM-D data. Thus, the formulated inks became easier to flow when GelMA was incorporated. This was consistent with employing lower printing pressure in printing the formulated CNF/ GelMA ink compared with CNF itself, which will be discussed later.

The decreased storage modulus of the inks (Figure 1d) with more GelMA was also revealed when the oscillation measurements were performed on the formulated bioinks. The bioink viscoelasticity was recorded for $12 \mathrm{~min}$ with UV turning on at 120 s. Immediately after UV irradiation was turned on, the storage modulus increased rapidly for all the ink formulations of CNF/GelMA followed by a level-off plateau, referred to the required cross-linking time. As shown in Figure 1d, the required time for all the formulated inks to reach full crosslinking was as short as within $30 \mathrm{~s}$. The rate of cross-linking is important for biofabrication that requires gelation during a very short time window, such as for in situ gelation during extrusion. ${ }^{44}$ For controls, measurements were also performed on $\mathrm{CNF}$ and $1.0 \%$ GelMA solution, and their viscoelasticity did not change upon UV irradiation, yet even the bioink of CNF:GelMA=5: 1 containing only $0.2 \mathrm{w} / \mathrm{v} \%$ GelMA displayed an apparent cross-linking phenomenon, as shown in Figure $1 \mathrm{~d}$. According to a recent study by O'Connell et al., no clear crosslinking phenomenon was observed when GelMA concentration was below $2.5 \mathrm{w} / \mathrm{v} \% .{ }^{44}$ This indirectly revealed that the presence of TEMPO-oxidized CNF could play a key role in facilitating cross-linking of low-concentration GelMA. As well, the gelation time for low-concentration GelMA in the presence of TEMPO-oxidized CNF is comparable with that of high concentration of GelMA solutions $(>5 \mathrm{w} / \mathrm{v} \%)$ in O'Connell et al.'s study ${ }^{44}$ when both $0.5 \mathrm{w} / \mathrm{v} \%$ concentration of photoinitiator Irgacure 2959 and $10 \mathrm{~mW} / \mathrm{cm}^{2} \mathrm{UV}$ intensity were applied. Therefore, it is hypothesized that the CNF fibers that supplied the matrix for the GelMA oriented in a more entangled fashion with the methacryloyls locally concentrated to facilitate the UV cross-linking, even at a rather low concentration of GelMA.
It is worth noting the different ink behaviors regarding the variety of starting CNFs. For example, in the previous studies by Markstedt et al, ${ }^{16,43}$ enzymatically and mechanically produced CNFs were used without supplying surface charge like in the case of TEMPO-oxidized CNF. First, the consistency of enzymatically and mechanically produced CNF can be a few times higher than that of TEMPO-oxidized $\mathrm{CNF}$, and the shape fidelity can be enhanced by the high matter load of the inks. Meanwhile, TEMPO-oxidized CNF prepared in our approach is only present at a comparatively low consistency, and this, to some extent, limits the matter load in the bioinks. However, in terms of printing resolution, TEMPO-oxidized CNF was superior with being successfully printed using the cylindrical steel tip $(200 \mu \mathrm{m})$, compared to a conical tip $(420 \mu \mathrm{m})$ for noncharged CNFs. ${ }^{16,43}$ More importantly, in the present study, the negatively charged CNF by TEMPO-mediated oxidation facilitated the UV crosslinking of low-concentration GelMA (less than $1.0 \mathrm{w} / \mathrm{v} \%$ ), possibly due to surface ionic interaction between CNF and GelMA, whereas in another study using mechanically grinded $\mathrm{CNF}$ as a rheology modifier and structural builder, $2 \mathrm{w} / \mathrm{v} \%$ CNF was required for $5 \mathrm{w} / \mathrm{v} \%$ GelMA printing. ${ }^{32}$

Bioink Printability. The printability and shape fidelity of the formulated bioinks were investigated by an extrusion-based $3 \mathrm{D}$ printer with a pneumatic dispensing system and cylindrical stainless-steel tips. The printed strut diameters are determined by three parameters: printing speed, extrusion pressure, and tip diameter. However, when the viscoelasticity of the bioinks changes, the printing parameters need to be adjusted accordingly. In order to reduce the complexity to standardize the printing parameter, the same printing speed of $2000 \mathrm{~mm} /$ min was applied throughout this study. A uniform and reproducible strut diameter of printed scaffolds was achieved by choosing appropriate dispensing tip diameters and tuning extrusion pressure in a limited range. Dispensing tips of $27 \mathrm{G}$ $(\varnothing 0.2 \mathrm{~mm})$ were used for the CNF bioink, and tips of $30 \mathrm{G}(\varnothing$ $0.15 \mathrm{~mm}$ ) were used for CNF/GelMA bioinks. By tuning the pneumatic pressure between 60 and $80 \mathrm{kPa}$, the extruded strut diameter was controlled at $0.25 \pm 0.01 \mathrm{~mm}$. The screened parameters are listed in Table 1 . Moreover, the diameter of dispensing tips was adjusted from 0.2 to $0.15 \mathrm{~mm}$ when the ink was changed from CNF to CNF/GelMA inks, which was consistent with the decreased storage modulus of $\mathrm{CNF} /$ GelMA inks as discussed previously. This is because the adsorption of GelMA on CNF has an impact on the viscoelasticity of the formulated ink hydrogel and makes the flow through the needle easier for the CNF/GelMA ink, as the decreased zero-shear viscosity indicated in Figure 1c.

Printability and shape fidelity are not only dependent on the printing parameters but also determined by the solid content of the bioinks, as well as cross-linking treatments during and after printing. The incorporated GelMA increased the solid content in the bioinks and supplied the secondary cross-linking network, which improved the shape fidelity. In the current study, the carboxylic groups on the CNF surface were utilized to supply instant ionic cross-linking by adding $5 \% \mathrm{CaCl}_{2}$ solution dropwise during printing. While the printed object was completed, the whole scaffold was subjected to UV irradiation to cross-link GelMA. Afterward, the scaffolds were stored in $\mathrm{PBS} / \mathrm{Ca}^{2+}$ solution for further study. $\mathrm{Ca}^{2+}$ ions temporally maintain the structural integrity of the printed constructs, avoiding collapse before UV cross-linking. This enabled the TEMPO-oxidized CNF to maintain the scaffold 

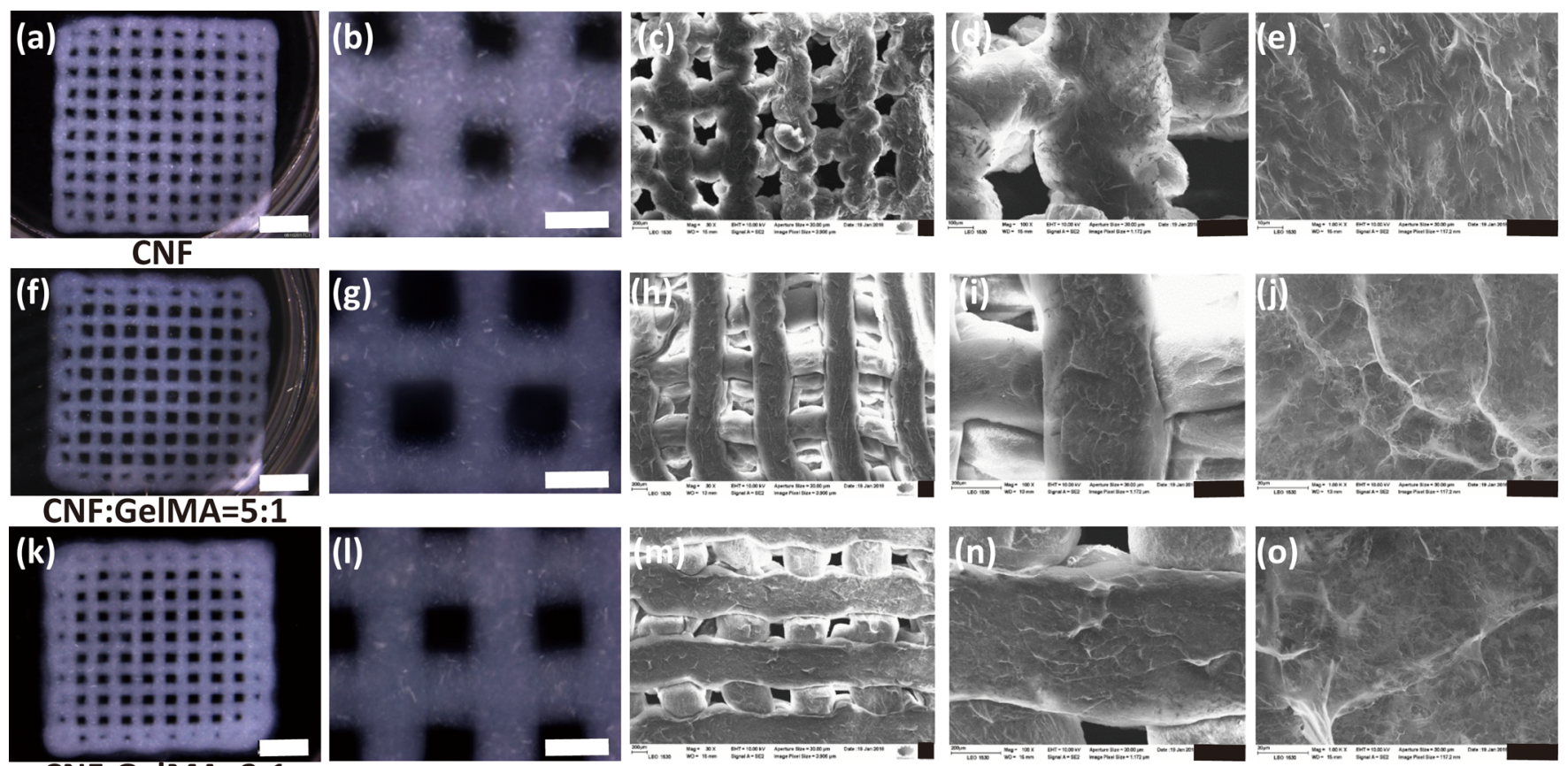

CNF:GelMA=2:1
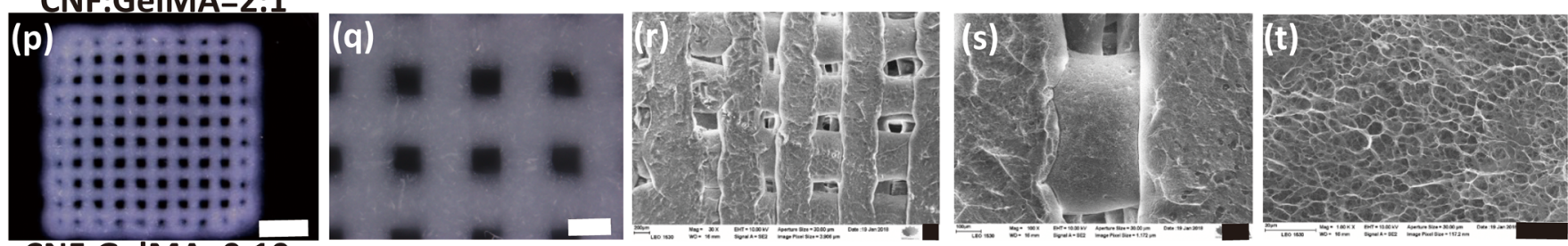

CNF:GelMA=9:10

Figure 2. Left two columns: optical microscopic images showing morphology of the printed scaffolds from the inks of CNF hydrogel (1.0 w/v \%) $(\mathrm{a}, \mathrm{b})$ and CNF/GelMA hydrogels with weight compositional ratios of 5:1 (f,g), 2:1 (k,1), and 9:10 (p,q). Right three columns: SEM images of the printed scaffolds from the inks of CNF hydrogel $(\mathrm{c}-\mathrm{e})$ and CNF/GelMA hydrogels with weight compositional ratios of 5:1 (h-j), 2:1 (m-o), and 9:10 (r-t). The images in the same column have the same scale bar with $2 \mathrm{~mm}(\mathrm{a}, \mathrm{f}, \mathrm{k}, \mathrm{p}), 500 \mu \mathrm{m}(\mathrm{b}, \mathrm{g}, \mathrm{l}, \mathrm{q}), 200 \mu \mathrm{m}(\mathrm{c}, \mathrm{h}, \mathrm{m}, \mathrm{r}), 100 \mu \mathrm{m}(\mathrm{d}, \mathrm{i}, \mathrm{n}, \mathrm{s})$, and $20 \mu \mathrm{m}(\mathrm{e}, \mathrm{j}, \mathrm{o}, \mathrm{t})$ from left to right column.

integrity at a low concentration of $1 \mathrm{w} / \mathrm{v} \%$ instead of using a high concentration, that is, above $2.5 \mathrm{wt} \%$ dry consistency for the mechanically grinded $\mathrm{CNF}^{43}$ Meanwhile, as a facile approach, the rapid cross-linking (as shown in Figure 1d) upon UV irradiation works more conveniently compared with introducing a high dosage of chemicals for cross-linking. ${ }^{32}$

Scaffold Morphology. One of the goals of this study was to develop printable inks, which could be printed with excellent 3D shape fidelity and long shelf-time stability. Therefore, the shape fidelity of the printed scaffolds under physiological conditions was assessed with optical microscopy imaging in $\mathrm{PBS} / \mathrm{Ca}^{2+}$ buffer. As shown in Figure 2, scaffolds in high resolution were observed with an excellent dimensional stability. Adding GelMA into ink formulations made the printed struts with clear and smooth boundaries when compared with CNF only, as shown in the second column of Figure 2. The well-defined struts benefited from the possibility of using extra fine tip diameters due to the excellent rheological behaviors of these low-concentration bioinks. In addition, the surface charge of CNF made it possible for instantaneous $\mathrm{Ca}^{2+}$ cross-linking to keep shape fidelity.

Similarly, as shown by the SEM images in Figure 2, the freeze-dried scaffolds of CNF/GelMA showed different surface morphologies from the CNF scaffolds. After drying, the CNF scaffolds did not straightly align in their originally printed form, and the pores between the struts in the scaffolds became deformed without keeping the resolution as it is in hydrogel form. From high-magnification images of the CNF scaffolds (Figure 2d,e), large CNF fiber bundles and aggregates could be observed, which is due to the strong hydrogen bonding after freeze-drying. For the dried scaffolds of CNF/GelMA, the struts kept the alignments better than the CNF scaffolds. As shown in the high-magnification images (Figure 2i,n,s,), the surface of the struts showed a smoother morphology than that of the CNF scaffolds (Figure 2d). Moreover, the incorporated GelMA gives more networks with increasing content of GelMA, as shown in Figure 2j,o,t, which is distinctive from the CNF scaffolds where only fiber bundles are observed on the surface (Figure 2e). The adsorption of GelMA onto CNF hinders the formation of hydrogen bonding between $\mathrm{CNF}$ fibers, which further has a significant impact on guiding the fiber orientation. ${ }^{32}$ In the sample of CNF:GelMA=9:10, the strut surface showed a microporous structure, which is considered beneficial for cell attachment and proliferation during in vivo conditions by improving diffusion rates to and from the scaffolds. ${ }^{45}$

The average diameter of the printed struts increased by 1 fold after swelling in PBS buffer compared with each theoretical diameter as listed in Table 1 . The scaffolds printed with CNF:GelMA=5:1 and 2:1 had average strut diameters smaller than that of CNF scaffolds, indicating less swelling of printed struts, which is possibly due to the cross-linked GelMA 
restricting the swelling of the strut. However, the strut diameter increased with increasing GelMA content, with CNF:GelMA=9:10 reaching approximately $0.6 \mathrm{~mm}$, which is similar to the strut diameter of CNF. Although this was contrary to its cross-linking degree, it might be due to the disruption of GelMA to the alignment and bonding between CNF fibers, which counteracted the restriction from GelMA cross-linking.

Rehydration Property. The rehydration kinetics, otherwise termed swelling ratio, is a crucial factor that needs consideration for the biomedical application of hydrogel scaffolds. The rehydration possibility directly refers to their hydration ability and stability and indirectly reveals their mechanical stiffness and structural properties. ${ }^{33,46}$ The swelling of the freeze-dried scaffolds, maintaining a moist environment, is beneficial for wound healing. ${ }^{47}$ The time-dependent swelling profiles were recorded by immersing the freeze-dried scaffolds with Milli-Q water, as shown in Figure 3. The CNF aerogels

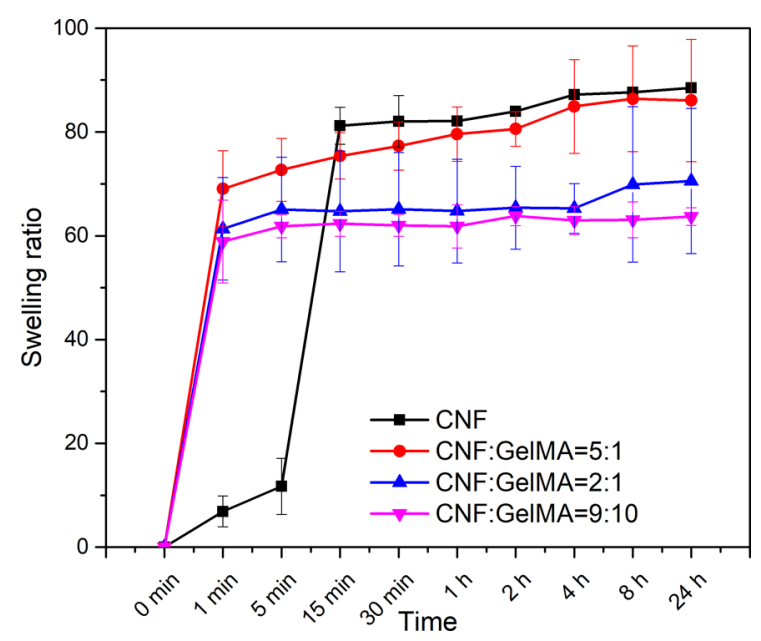

Figure 3. Water uptake behavior of the freeze-dried scaffolds against time scale. Swelling ratio of liquid $\mathrm{N}_{2}$ frozen and then freeze-dried scaffolds printed from the inks of CNF hydrogel and CNF/GelMA hydrogels with weight compositional ratios of 5:1, 2:1, and 9:10. The values were recorded within a period of $24 \mathrm{~h}$.

made of TEMPO-mediated oxidation can uptake water up to around 90 times its own weight, which is in line with what is reported in our previous study. ${ }^{38}$ Overall, the rehydration equilibrium was restricted by the cross-linking of incorporated GelMA. Meanwhile, the rehydration kinetics behaved differently between CNF scaffolds and CNF/GelMA scaffolds. The CNF/GelMA scaffolds showed rapid rehydration ability within the first minute, with the scaffolds of CNF:GelMA=9:10 reaching equilibrium within $5 \mathrm{~min}$. The rehydration kinetics of CNF:GelMA=2:1 and 5:1 scaffolds showed two-step curves: after rapid rehydration at $1 \mathrm{~min}$, the swelling rate slowed down and reached equilibrium after $8 \mathrm{~h}$. However, the CNF scaffolds showed different kinetics. CNF scaffolds had a slow rehydration rate for the first $5 \mathrm{~min}$; then, a fast swelling rate resulted in absorption of around 90-fold water compared with their dry weight, followed by a much slower swelling rate and reaching equilibrium at around $4 \mathrm{~h}$. The three-step water adsorption curve of CNF scaffolds can be attributed to the strong hydrogen bonding between the CNF fibers, and it takes a longer time for water to penetrate the fiber bundles after freeze-drying. ${ }^{33,38}$ However, when a small amount of GelMA was introduced (CNF:GelMA=5:1 and 2:1), the rehydration kinetics changed to two-step curves. This likely resulted from the adsorption of GelMA firmly binding with the surface of CNF fibers, as QCM-D data indicated, which interrupted the hydrogen bonding between CNF fibers. This could also explain the lower rehydration ratio of scaffolds with more GelMA incorporated, since more hydrogen bonding sites were occupied by GelMA molecules. However, compared with CNF scaffolds, the incorporation of GelMA also made the scaffolds more accessible to water with a high swelling ratio at $1 \mathrm{~min}$. This could be explained by the porous topography of the GelMA incorporated scaffolds. The rapid rehydration kinetics could be a beneficial feature in regard to both loading therapeutic drugs and absorbing wound exudate in the use of the dried scaffold.

Mechanical Properties. Mechanical strength of scaffolds plays a significant role in their applications to meet the bioenvironmental needs. CNF, particularly prepared by TEMPO-mediated oxidation to introduce carboxylates, undergoes ionic interaction and even dissociation in aqueous media possessing high ionic strength. That will threaten the stability of mechanical properties of scaffolds. In our previous study, when CNF solely was printed from a relatively lower concentration $(\sim 0.7 \mathrm{wt} \%)$ hydrogel, the printed scaffolds dissociated into water. ${ }^{21}$ Thus, higher concentration of CNF hydrogel was demanded for printing, and a dual-cross-linking approach was developed to further increase the mechanical strength of the scaffolds. Here, GelMA was incorporated together with CNF to form a hydrogel, and its mechanical strength can be easily tuned by UV cross-linking. Mechanical properties of the formulated hydrogels were evaluated by the compression tests on both cast discs (Figure $4 \mathrm{a}$ ) and printed scaffolds. As shown in Figure $4 b$, the compressive Young's modulus indicated clearly that UV cross-linked GelMA condensed the structure and improved the rigidity of the hydrogel. Figure $4 \mathrm{c}$ shows the correlation between compressive Young's modulus and GelMA content for cast discs and printed scaffolds. The compressive Young's modulus of the cast discs varied from 2.3 to $4.5 \mathrm{kPa}$ as the content of GelMA increased. The compressive Young's modulus of CNF:Gel$\mathrm{MA}=9: 10$ disc samples increased by 1 -fold compared with that of the CNF discs. This could be attributed to the increased solid content load ${ }^{48}$ and cross-linked GelMA network. The increasing trend of compressive Young's modulus of the printed scaffolds followed that of cast discs. This is also relevant to the result of ink storage modulus after UV irradiation (Figure 1d): the $G^{\prime}$ value of the CNF/GelMA inks increased with addition of more GelMA, indicating the increased strength of formulated hydrogels. The difference of compressive Young's modulus between the discs and the scaffolds originated from the fractional porosity from a solid disc to a porous scaffold. It was reported that fibroblasts start to respond by spreading with the matrix at a stiffness range around $3 \mathrm{kPa}{ }^{49}$ Thus, the printed scaffolds with mechanical Young's modulus in the range of 2.5 to $5 \mathrm{kPa}$ could be suitable for wound healing application. ${ }^{50}$ Furthermore, the local surface stiffness of the printed scaffolds was monitored using AFM, as shown in Figure 4d. The surface Young's modulus followed the same trend as the compressive Young's modulus of both cast discs and printed scaffolds. The average surface Young's modulus increased from 400 to $700 \mathrm{~Pa}$ with increasing GelMA content. The surface stiffness of the printed scaffolds of CNF/ 


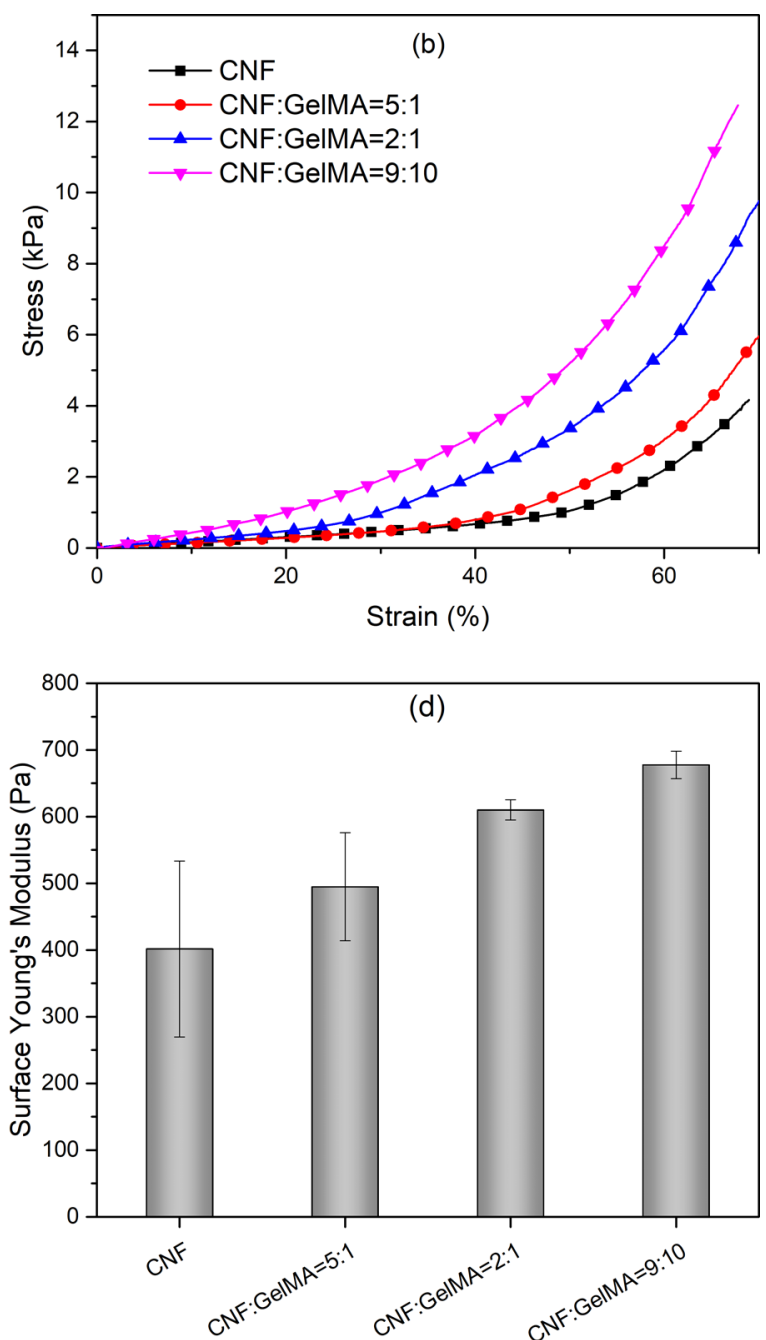

Figure 4. (a) Images of cast discs with formulated inks presenting from (i) to (iv) with hydrogels of CNF, CNF:GelMA=5:1, CNF:GelMA=2:1, and CNF:GelMA=9:10, respectively; (b) stress-strain plot of the formulated inks; (c) relevantly increased compressive Young's moduli among the cast discs (labeled as "Disc") and printed scaffolds (labeled as "Scaffold") after UV cross-linking; (d) tunable local surface stiffness with changing GelMA amounts in ink formulations measured by AFM measurements.

GelMA mainly corresponds to the cross-linking of various contents of GelMA incorporated in the scaffolds.

3D CNF Matrix Support Survival, Adhesion, and Proliferation of Fibroblast Cells. We next assessed the cytocompatibility of the 3D printed scaffolds of CNF/GelMA, a prerequisite for the material to be used for an ECMmimicking matrix. First, we tested the functionality of the 3D printed matrix in supporting cell attachment and survival. Mouse 3T3 fibroblasts, a key cell component involved in wound healing, were employed in the study. The cell adhesion capability was measured using a crystal violet assay after $12 \mathrm{~h}$ of cell incubation in the presence of the CNF matrix. It was found that, in comparison with $2 \mathrm{D}$ control coverslip samples, cells adhered slightly less on the CNF matrix, whereas the incorporation of GelMA encouraged cell adhesion (Figure 5a). This is likely due to the incorporated GelMA containing motifs like arginine-glycine-aspartic acid (RGD) sequences that could promote cell attachment. $^{24,51}$ In addition, after $24 \mathrm{~h}$ of cell incubation in the 3D matrix, cell samples were examined with the MTT colorimetric assay to quantitatively measure the capability of viable cells in metabolizing a dye [3-(4,5dimethylthiozol-2-yl)-2,5-diphenyl tetrazolium bromide].
These results clearly demonstrated that the $3 \mathrm{D}$ printed scaffolds of CNF/GelMA had no adverse effect on cell viability (Figure $5 \mathrm{~b}$ ). This is consistent with our previous studies regarding the cytotoxicity test of TEMPO-oxidized CNF that is well compatible with epithelial-derived HeLa cells and hematopoietic-derived Jurkat cells. ${ }^{33,38}$ Meanwhile, the cell viability tests demonstrated that the current material process was also friendly to mouse fibroblasts.

Moreover, we found that the 3D matrix supports cell proliferation in a longer incubation period with the verification of cell growth into the 3D matrix (Figures S1 and S2). This agreed with the finding of the positive effect of CNF/GelMA on cell proliferation as reported by Shin et al. ${ }^{32}$ The results showed that after 3 days of post cell seeding, there were twice the number of cells on samples of CNF:GelMA=5:1 compared with CNF scaffolds (Figure 6). Interestingly, higher contents of GelMA in samples of CNF:GelMA=2:1 and CNF:Gel$M A=9: 10$ had a weaker effect on enhancing cell proliferation. This dose-dependent correlation between the proliferative activity of the fibroblasts and the GelMA content suggests the importance of controlling GelMA concentration in producing such a matrix for potent fibroblast proliferation and further 
(a)

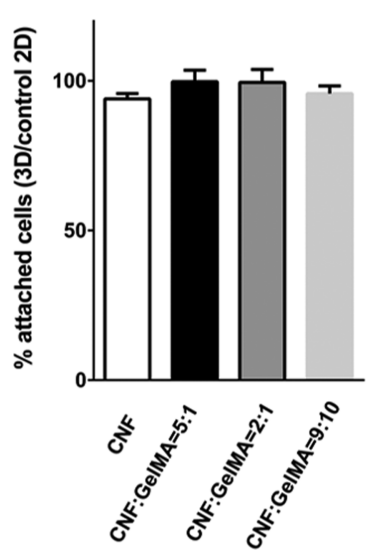

(b)

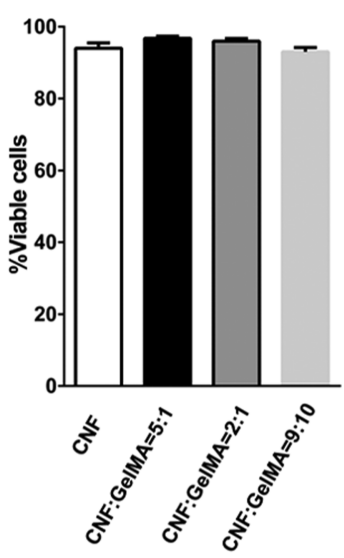

Figure 5. 3T 3 cells were incubated with the indicated 3D matrix in a density of $1 \times 10^{5}$ cells per well. (a) The cell adhesion was measured after $12 \mathrm{~h}$ of incubation using the crystal violet assay. (b) The cell survival rate was measured after $24 \mathrm{~h}$ of incubation by using the MTT assay. Bar $=$ mean \pm STDEV; $n=4$. Matrix hydrogels presenting inks of CNF and CNF/GelMA with weight compositional ratios of 5:1, 2:1, and 9:10.

(a)

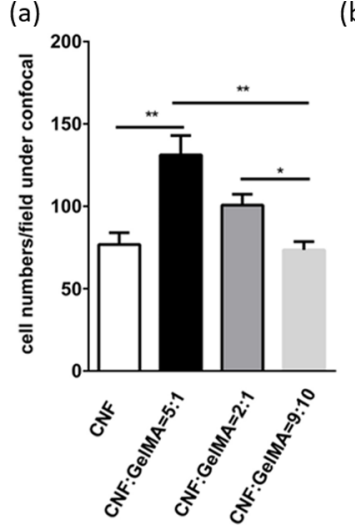

(b)


CNF:GelMA=5:1

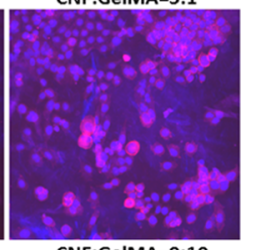

CNF:GelMA=9:10

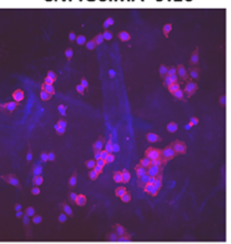

Figure 6. 3T 3 cells were incubated with the indicated $3 \mathrm{D}$ matrix in a density of $1 \times 10^{5}$ cells per well. The cell proliferation (a) and representative confocal images (b) were measured after 3 days of incubation. Scale bar, $50 \mu \mathrm{m}$. Bar = mean \pm STDEV; $n=4$. $^{*}, p<0.1$; $* *, p<0.01$. Matrix hydrogels presenting inks of CNF and CNF/ GelMA with weight compositional ratios of 5:1, 2:1, and 9:10.

prompt wound healing (Figure 6). As demonstrated by our recent research, ${ }^{21}$ when ink formulations of the CNF single component were used, the proliferation of fibroblasts was mainly regulated by the increased mechanical stiffness in the CNF hydrogel. However, in this study, the incorporation of GelMA influenced both the ink properties and the formed hydrogels, including cell adhesion motifs (i.e., RGDs), modulus, topographical features, and porosity, which have to be considered overall for cell migration and proliferation. In particular, the fibroblast proliferation in the matrix of CNF:GelMA=9:10 was not promoted as much as in the matrix of CNF:GelMA=5:1, although the matrix of CNF:Gel$\mathrm{MA}=9: 10$ demonstrated the highest surface stiffness among these four types of printed constructs. Thus, multiple factors including both tunable mechanical stiffness and the topographical morphology of printed CNF/GelMA should be accounted for regarding the proliferative activity of $3 \mathrm{~T} 3$ fibroblasts in these biomaterials. As displayed in Figure 2, the

topographical features became more porous on the printed struts of CNF/GelMA as the content of GelMA increased. The wall-separated microspores in the network on the strut surface of CNF:GelMA=9:10 were around 5-20 $\mu \mathrm{m}$ as measured in the sample after freeze-drying (Figure 2t). After swelling with water uptake, these pore dimensions are anticipated to be almost comparable to the size of mouse 3T3 fibroblast cells $(50-150 \mu \mathrm{m})$. It is suspected that the wall-like morphology might interfere with the cell migration if the fibroblasts could likely be entrapped in the network. ${ }^{52}$

In summary, the nontoxicity and the promoted proliferative activity of 3T3 fibroblasts in the CNF/GelMA scaffolds provide good opportunities for their safe application in wound healing therapy.

\section{CONCLUSIONS}

This study presents a novel low-concentration ink formulation based on $1.0 \mathrm{w} / \mathrm{v} \%$ TEMPO-oxidized CNF and up to $1.0 \mathrm{w} / \mathrm{v}$ $\%$ GelMA for extrusion-based 3D printing of nanocellulose hydrogel scaffolds. The molecular interaction between these two biopolymers of TEMPO-oxidized CNF and GelMA modified the rheological properties of the CNF. More importantly, the presence of TEMPO-oxidized CNF facilitated the UV cross-linking of GelMA incorporated in ultralow concentrations in the formulated bioinks. By direct ink writing assisted by UV postcuring, high-resolution scaffolds of CNF/ GelMA were successfully printed, and these scaffolds demonstrated high fidelity and stability. By tuning the compositional ratio between CNF and GelMA, the compressive Young's modulus and local surface stiffness could be well tuned. The developed ink formulations are noncytotoxic and cytocompatible with mouse 3T3 fibroblasts. Furthermore, ink formulations of CNF incorporated with GelMA, particularly with CNF/GelMA ratios of 2:1 and 5:1, promoted the proliferative activity of $3 \mathrm{~T} 3$ fibroblasts when compared with the plain CNF hydrogel. Owing to the influences of both surface stiffness and topographical features in the biomaterials, these scaffolds of CNF:GelMA=5:1 showed outstanding performance on promoting fibroblast proliferation. In summary, the developed low-concentration ink formulations of CNF/GelMA present a facile yet effective approach to fabricate hydrogel scaffolds with tunable mechanical strength that show great potential toward such applications as wound healing and soft tissue regeneration.

\section{ASSOCIATED CONTENT}

\section{Supporting Information}

The Supporting Information is available free of charge on the ACS Publications website at DOI: 10.1021/acsami.8b21268.

Four stacks of confocal images and maximal 3D projection of confocal images on the Z-stack microscopic 3D image showing the adhered cells inside the 3D printed matrix (PDF)

\section{AUTHOR INFORMATION}

\section{Corresponding Authors}

*E-mail: cxu@abo.fi (C.X.).

*E-mail: gwallace@uow.edu.au (G.G.W.).

ORCID $\odot$

Wenyang Xu: 0000-0002-3205-7891

Paul J. Molino: 0000-0002-8868-2733 
Chunlin Xu: 0000-0003-1860-9669

Gordon G. Wallace: 0000-0002-0381-7273

\section{Author Contributions}

${ }^{\#}$ W.X. and B.Z.M. equally contributed to the present work.

\section{Notes}

The authors declare no competing financial interest.

\section{ACKNOWLEDGMENTS}

W.X. would like to thank the Johan Gadolin Process Chemistry Centre of Åbo Akademi University for their funding, the Harry Elvings legat and Finnish Paper Engineers's Association for travel grants, and funding from the ARC Centre of Excellence for Electromaterials Science (CE140100012) during a stay at the University of Wollongong. X.W. and C.X. would like to thank the Academy of Finland (298325) for funding. Authors would like to thank the Australian National Fabrication Facility (ANFF) Material Node for the use of equipment and the supply of GelMA by Dr. Sanjeev Gambhir. Authors would like to acknowledge the use of the facilities and assistance from Mr. Tony Romeo at the University of Wollongong Electron Microscopy Centre. Dr. Sepidar Sayyar and doctoral candidates Yuchao Fan and Jianfeng Li at IPRI, UOW are acknowledged for the help and discussion with practical experiments. F. C. would like to thank Sigrid Jusélius foundation, the National Natural Science Foundation of China (Grant no. 81702750) and the Basic Research Project of Shenzhen (Grant no. JCY20170818164756460) for funding the cell tests.

\section{REFERENCES}

(1) Barry, R. A., III; Shepherd, R. F.; Hanson, J. N.; Nuzzo, R. G.; Wiltzius, P.; Lewis, J. A. Direct-Write Assembly of 3D Hydrogel Scaffolds for Guided Cell Growth. Adv. Mater. 2009, 21, 2407-2410.

(2) Murphy, S. V.; Atala, A. 3D bioprinting of Tissues and Organs. Nat. Biotechnol. 2014, 32, 773.

(3) Siqueira, G.; Kokkinis, D.; Libanori, R.; Hausmann, M. K.; Gladman, A. S.; Neels, A.; Tingaut, P.; Zimmermann, T.; Lewis, J. A.; Studart, A. R. Cellulose Nanocrystal Inks for 3D Printing of Textured Cellular Architectures. Adv. Funct. Mater. 2017, 27, 1604619.

(4) Palaganas, N. B.; Mangadlao, J. D.; de Leon, A. C. C.; Palaganas, J. O.; Pangilinan, K. D.; Lee, Y. J.; Advincula, R. C. 3D Printing of Photocurable Cellulose Nanocrystal Composite for Fabrication of Complex Architectures via Stereolithography. ACS Appl. Mater. Interfaces 2017, 9, 34314-34324.

(5) Jia, C.; Bian, H.; Gao, T.; Jiang, F.; Kierzewski, I. M.; Wang, Y.; Yao, Y.; Chen, L.; Shao, Z.; Zhu, J. Y.; Hu, L. Thermally Stable Cellulose Nanocrystals toward High-Performance 2D and 3D Nanostructures. ACS Appl. Mater. Interfaces 2017, 9, 28922-28929.

(6) Li, V. C.-F.; Dunn, C. K.; Zhang, Z.; Deng, Y.; Qi, H. J. Direct Ink Write (DIW) 3D Printed Cellulose Nanocrystal Aerogel Structures. Sci. Rep. 2017, 7, 8018.

(7) Sultan, S.; Mathew, A. P. 3D printed Scaffolds with Gradient Porosity Based on A Cellulose Nanocrystal Hydrogel. Nanoscale 2018, $10,4421-4431$.

(8) Chinga-Carrasco, G. Potential and Limitations of Nanocelluloses as Components in Biocomposite Inks for Three-Dimensional Bioprinting and for Biomedical Devices. Biomacromolecules 2018, 19, 701-711.

(9) Xu, W.; Wang, X.; Sandler, N.; Willför, S.; Xu, C. ThreeDimensional Printing of Wood-Derived Biopolymers: A review focused on Biomedical Applications. ACS Sustainable Chem. Eng. 2018, 6, 5663-5680.

(10) Avila, H. M.; Schwarz, S.; Rotter, N.; Gatenholm, P. 3D Bioprinting of Human Chondrocyte-Laden Nanocellulose Hydrogels for Patient-Specific Auricular Cartilage Regeneration. Bioprinting 2016, 1, 22-35.

(11) Gatenholm, P.; Backdahl, H.; Tzavaras, T. J.; Davalos, R. V.; Sano, M. B. Three-Dimensional Bioprinting of Biosynthetic Cellulose (BC) Implants and Scaffolds for Tissue Engineering. U.S. Patent 8,691,974, April 8, 2014.

(12) Håkansson, K. M. O.; Henriksson, I. C.; de la Peña Vázquez, C.; Kuzmenko, V.; Markstedt, K.; Enoksson, P.; Gatenholm, P. Solidification of 3D Printed Nanofibril Hydrogels into Functional 3D Cellulose Structures. Adv. Mater. Technol. 2016, 1, 1600096.

(13) Henriksson, I.; Gatenholm, P.; Hägg, D. A. Increased Lipid Accumulation and Adipogenic Gene Expression of Adipocytes in 3D Bioprinted Nanocellulose Scaffolds. Biofabrication 2017, 9, No. 015022.

(14) Möller, T.; Amoroso, M.; Hägg, D.; Brantsing, C.; Rotter, N.; Apelgren, P.; Lindahl, A.; Kölby, L.; Gatenholm, P. In Vivo Chondrogenesis in 3D Bioprinted Human Cell-Laden Hydrogel Constructs. Plast. Reconstr. Surg. Glob. Open 2017, 5, No. e1227.

(15) Müller, M.; Öztürk, E.; Arlov, Ø.; Gatenholm, P.; ZenobiWong, M. Alginate Sulfate-Nanocellulose Bioinks for Cartilage Bioprinting Applications. Ann. Biomed. Eng. 2017, 45, 210-223.

(16) Markstedt, K.; Mantas, A.; Tournier, I.; Martínez Ávila, H.; Hägg, D.; Gatenholm, P. 3D Bioprinting Human Chondrocytes with Nanocellulose-Alginate Bioink for Cartilage Tissue Engineering Applications. Biomacromolecules 2015, 16, 1489-1496.

(17) Hubbe, M. A.; Tayeb, P.; Joyce, M.; Tyagi, P.; Kehoe, M.; Dimic-Misic, K.; Pal, L. Rheology of Nanocellulose-rich Aqueous Suspensions: A Review. BioResources 2017, 12, 9556-9661.

(18) Kyle, S.; Jessop, Z. M.; Al-Sabah, A.; Whitaker, I. S. 'Printability'of Candidate Biomaterials for Extrusion Based 3d Printing: State-of-the-art. Adv. Healthcare Mater. 2017, 6, 1700264.

(19) Mandrycky, C.; Wang, Z.; Kim, K.; Kim, D.-H. 3D Bioprinting for Engineering Complex Tissues. Biotechnol. Adv. 2016, 34, 422434.

(20) Rees, A.; Powell, L. C.; Chinga-Carrasco, G.; Gethin, D. T.; Syverud, K.; Hill, K. E.; Thomas, D. W. 3D Bioprinting of Carboxymethylated-Periodate Oxidized Nanocellulose Constructs for Wound Dressing Applications. BioMed Res. Int. 2015, 2015, 925757.

(21) Xu, C.; Molino, B. Z.; Wang, X.; Cheng, F.; Xu, W.; Molino, P.; Bacher, M.; Su, D.; Rosenau, T.; Willfor, S.; Wallace, G. 3D printing of Nanocellulose Hydrogel Scaffolds with Tunable Mechanical Strength towards Wound Healing Application. J. Mater. Chem. B 2018, 6, 7066-7075.

(22) Torres-Rendon, J. G.; Femmer, T.; De Laporte, L.; Tigges, T.; Rahimi, K.; Gremse, F.; Zafarnia, S.; Lederle, W.; Ifuku, S.; Wessling, M.; Hardy, J. G.; Walther, A. Bioactive Gyroid Scaffolds formed by Sacrificial Templating of Nanocellulose and Nanochitin Hydrogels as Instructive Platforms for Biomimetic Tissue Engineering. Adv. Mater. 2015, 27, 2989-2995.

(23) Liu, Y.; Chan-Park, M. B. A Biomimetic Hydrogel based on Methacrylated Dextran-Graft-Lysine and Gelatin for 3D Smooth Muscle Cell Culture. Biomaterials 2010, 31, 1158-1170.

(24) Yue, K.; Trujillo-de Santiago, G.; Alvarez, M. M.; Tamayol, A.; Annabi, N.; Khademhosseini, A. Synthesis, Properties, and Biomedical Applications of Gelatin Methacryloyl (GelMA) Hydrogels. Biomaterials 2015, 73, 254-271.

(25) Zhao, Y.; Yao, R.; Ouyang, L.; Ding, H.; Zhang, T.; Zhang, K.; Cheng, S.; Sun, W. Three-Dimensional Printing of Hela Cells for Cervical Tumor Model In Vitro. Biofabrication 2014, 6, No. 035001.

(26) Stratesteffen, H.; Köpf, M.; Kreimendahl, F.; Blaeser, A.; Jockenhoevel, S.; Fischer, H. GelMA-Collagen Blends Enable Dropon-demand 3D Printablility and Promote Angiogenesis. Biofabrication 2017, 9, No. 045002.

(27) Sakai, S.; Ohi, H.; Hotta, T.; Kamei, H.; Taya, M. Differentiation Potential of Human Adipose Stem Cells Bioprinted with Hyaluronic Acid/Gelatin-based Bioink through Microextrusion and Visible Light-Initiated Crosslinking. Biopolymers 2018, 109, No. e23080. 
(28) Billiet, T.; Gevaert, E.; De Schryver, T.; Cornelissen, M.; Dubruel, P. The 3D printing of Gelatin Methacrylamide Cell-Laden Tissue-Engineered Constructs with High Cell Viability. Biomaterials 2014, 35, 49-62.

(29) Liu, W.; Zhang, Y. S.; Heinrich, M. A.; De Ferrari, F.; Jang, H. L.; Bakht, S. M.; Alvarez, M. M.; Yang, J.; Li, Y.-C.; Trujillo-de Santiago, G.; Miri, A. K.; Zhu, K.; Khoshakhlagh, P.; Prakash, G.; Cheng, H.; Guan, X.; Zhong, Z.; Ju, J.; Zhu, G. H.; Jin, X.; Shin, S. R.; Dokmeci, M. R.; Khademhosseini, A. Rapid Continuous Multimaterial Extrusion Bioprinting. Adv. Mater. 2017, 29, 1604630.

(30) Bertassoni, L. E.; Cardoso, J. C.; Manoharan, V.; Cristino, A. L.; Bhise, N. S.; Araujo, W. A.; Zorlutuna, P.; Vrana, N. E.; Ghaemmaghami, A. M.; Dokmeci, M. R.; Khademhosseini, A. Direct-Write Bioprinting of Cell-Laden Methacrylated Gelatin Hydrogels. Biofabrication 2014, 6, No. 024105.

(31) Yin, J.; Yan, M.; Wang, Y.; Fu, J.; Suo, H. 3D Bioprinting of Low-Concentration Cell-Laden Gelatin Methacrylate (GelMA) Bioinks with A Two-Step Cross-linking Strategy. ACS Appl. Mater. Interfaces 2018, 10, 6849-6857.

(32) Shin, S.; Park, S.; Park, M.; Jeong, E.; Na, K.; Youn, H. J.; Hyun, J. Cellulose Nanofibers for the Enhancement of Printability of Low Viscosity Gelatin Derivatives. BioResources 2017, 12, 2941-2954.

(33) Liu, J.; Chinga-Carrasco, G.; Cheng, F.; Xu, W.; Willför, S.; Syverud, K.; Xu, C. Hemicellulose-Reinforced Nanocellulose Hydrogels for Wound Healing Application. Cellulose 2016, 23, 3129-3143.

(34) Costantini, M.; Testa, S.; Fornetti, E.; Barbetta, A.; Trombetta, M.; Cannata, S. M.; Gargioli, C.; Rainer, A. Engineering Muscle Networks in 3D Gelatin Methacryloyl Hydrogels: Influence of Mechanical Stiffness and Geometrical Confinement. Front. Bioeng. Biotechnol. 2017, 5, 22.

(35) Valle-Delgado, J. J.; Johansson, L.-S.; Österberg, M. Bioinspired Lubricating Films of Cellulose Nanofibrils and Hyaluronic Acid. Colloids Surf., B 2016, 138, 86-93.

(36) Eronen, P.; Österberg, M.; Heikkinen, S.; Tenkanen, M.; Laine, J. Interactions of Structurally Different Hemicelluloses with Nanofibrillar Cellulose. Carbohydr. Polym. 2011, 86, 1281-1290.

(37) Höök, F.; Kasemo, B.; Nylander, T.; Fant, C.; Sott, K.; Elwing, H. Variations in Coupled Water, Viscoelastic Properties, and Film Thickness of A Mefp-1 Protein Film During Adsorption and Crosslinking: A Quartz Crystal Microbalance with Dissipation Monitoring, Ellipsometry, and Surface Plasmon Resonance Study. Anal. Chem. 2001, 73, 5796-5804.

(38) Liu, J.; Cheng, F.; Grënman, H.; Spoljaric, S.; Seppälä, J.; Eriksson, J. E.; Willför, S.; Xu, C. Development of Nanocellulose Scaffolds with Tunable Structures to Support 3D Cell Culture. Carbohydr. Polym. 2016, 148, 259-271.

(39) Jia, W.; Gungor-Ozkerim, P. S.; Zhang, Y. S.; Yue, K.; Zhu, K.; Liu, W.; Pi, Q.; Byambaa, B.; Dokmeci, M. R.; Shin, S. R.; Khademhosseini, A. Direct 3D Bioprinting of Perfusable Vascular Constructs Using A Blend Bioink. Biomaterials 2016, 106, 58-68.

(40) Wang, H.; Hansen, M. B.; Löwik, D. W. P. M.; van Hest, J. C. M.; Li, Y.; Jansen, J. A.; Leeuwenburgh, S. C. G. Oppositely Charged Gelatin Nanospheres as Building Blocks for Injectable and Biodegradable Gels. Adv. Mater. 2011, 23, H119-H124.

(41) Shirahama, H.; Lee, B. H.; Tan, L. P.; Cho, N.-J. Precise Tuning of Facile One-pot Gelatin Methacryloyl (GeLMA) Synthesis. Sci. Rep. 2016, 6, 31036.

(42) Lozhechnikova, A.; Dax, D.; Vartiainen, J.; Willför, S.; Xu, C.; Österberg, M. Modification of Nanofibrillated Cellulose Using Amphiphilic Block-Structured Galactoglucomannans. Carbohydr. Polym. 2014, 110, 163-172.

(43) Markstedt, K.; Escalante, A.; Toriz, G.; Gatenholm, P. Biomimetic Inks Based on Cellulose Nanofibrils and Cross-Linkable Xylans for 3D Printing. ACS Appl. Mater. Interfaces 2017, 9, 4087840886.

(44) O'Connell, C. D.; Zhang, B.; Onofrillo, C.; Duchi, S.; Blanchard, R.; Quigley, A.; Bourke, J.; Gambhir, S.; Kapsa, R.; Di Bella, C.; Choong, P.; Wallace, G. G. Tailoring the Mechanical Properties of Gelatin Methacryloyl Hydrogels through Manipulation of the Photocrosslinking Conditions. Soft Matter 2018, 14, 21422151.

(45) Naseri, N.; Poirier, J.-M.; Girandon, L.; Fröhlich, M.; Oksman, K.; Mathew, A. P. 3-Dimensional Porous Nanocomposite Scaffolds Based on Cellulose Nanofibers for Cartilage Tissue Engineering: Tailoring of Porosity and Mechanical Performance. RSC Adv. 2016, 6, 5999-6007.

(46) Barbucci, H. Biological Properties and Applications; Springer: New York, 2009.

(47) Holback, H.; Yeo, Y.; Park, K. Hydrogel Swelling Behavior and its Biomedical Applications. In Biomedical Hydrogels; Elsevier: Amsterdam, 2011; pp 3-24.

(48) Leppiniemi, J.; Lahtinen, P.; Paajanen, A.; Mahlberg, R.; MetsäKortelainen, S.; Pinomaa, T.; Pajari, H.; Vikholm-Lundin, I.; Pursula, P.; Hytönen, V. P. 3D-Printable Bioactivated Nanocellulose-Alginate Hydrogels. ACS Appl. Mater. Interfaces 2017, 9, 21959-21970.

(49) Yeung, T.; Georges, P. C.; Flanagan, L. A.; Marg, B.; Ortiz, M.; Funaki, M.; Zahir, N.; Ming, W.; Weaver, V.; Janmey, P. A. Effects of Substrate Stiffness on Cell Morphology, Cytoskeletal Structure, and Adhesion. Cytoskeleton 2005, 60, 24-34.

(50) Janmey, P. A.; Miller, R. T. Mechanisms of Mechanical Signaling in Development and Disease. J. Cell. Sci. 2011, 124, 9-18.

(51) Nichol, J. W.; Koshy, S. T.; Bae, H.; Hwang, C. M.; Yamanlar, S.; Khademhosseini, A. Cell-Laden Microengineered Gelatin Methacrylate Hydrogels. Biomaterials 2010, 31, 5536-5544.

(52) Murphy, C. M.; O'Brien, F. J. Understanding the Effect of Mean Pore Size on Cell Activity in Collagen-Glycosaminoglycan Scaffolds. Cell Adhes. Migr. 2010, 4, 377-381. 